\title{
ПРОДОЛЬНОЕ ПЕРЕМЕШИВАНИЕ И ЗАДЕРЖКА ЖИДКОСТИ В НАСАДОЧНОЙ КОЛОННЕ ПРИ МАЛОИ СКОРОСТИ ГАЗОВОГО ПОТОКА
}

Структура математической модели любого массообменного или химического процесса, протекающего в насадочной колонне, определяется прежде всего гидродинамическими параметрами, в том числе продольным перемешиванием жидкости и газа, а также временем пребывания частиц потоков в колонне. В некоторых химических и массообменных процессах, имеющих место между газом и жидкостью, например, при присоединении хлористого водорода к жидким алкадиенам в гидрохлораторах типа насадочной колонны, скорость газа практически равняется нулю. Для математического моделирования таких процессов необходимо установить количественные зависимости, выражающие влияние различных физикохимических факторов на продольное перемешивание и задержку жидкости в колонне в условиях, когда скорость газа практически равна нулю. С этой целью нами были проведены опыты на лабораторной установке. Справедливость полученных экспериментальных зависимостей проверялась на полупромышленном гидрохлораторе.

Лабораторные опыты проводились в стеклянной колонне диаметром 25 м. и высотой насадки 1,0 м. В качестве насадки служили стеклянные кольца Рашига размером $4,5 \times 4,5 \times 0,7$ мм. Опыты на полупромышленной установке проводились в гидрохлораторе типа насадочной колонны диаметром 200 мм и высотой насадки 3,5 м. Насадка (кольца Рашига размером $16 \times 16 \times 4$ м. ) была изготовлена из АТM. В качестве жидкой фазы в обоих случаях применялись смеси изопрена и его гидрохлоридов (2-хлор-2-метилбутен-3, 1-хлор-3-метилбутен-2), кроме того, в лабораторных опытах для установления влияния. поверхностного натяжения смесь этилового спирта с водой. Газовой фазой в лабораторных опытах служил воздух, а в опытах на полупромышленном гидрохлораторе сухой хлористый водород. Кроме основного агента газовая фаза содержала пары жидкости.

На вход жидкости в колонну при разных стационарных режимах было наложено возмущение в виде дельта-функций (импульсный ввод индикатора). В результате возмущения получалась функция распределения индикатора по времени, или т. н. $C$-выходная кривая. В качестве индикатора в опытах с изопреном и его гидрохлоридами использовался кубовый голубой К (индантреновый синий БЦ). Этот краситель оказался хорошо растворимым в углеводородах, под действием хлористого водорода интен- 
сивность его окраски не изменялась. Концентрация индикатора в пробах, взятых через равные интервалы из выходного потока, определялась колориметрически. В опытах со смесями, состоящими из воды и этилового спирта, индикатором служил хлористый натрий, концентрация которого определялась аргентометрически. На лабораторной установке проведено 45 , а на полупромышленном гидрохлораторе -7 опытов.

В ходе статистической обработки полученных результатов в виде моментов распределения первого и второго порядков определены среднее время пребывания частиц жидкости в колонне $\tau$ и дисперсия времени пребывания в безразмерных единицах $\sigma^{2}$.

Гидродинамика потока жидкой фазы в насадочной колонне обычно представляется однопараметрической диффузионной моделью, которая в безразмерном виде выражается уравнением:

$$
-\frac{d C}{d z}+\frac{1}{P e} \cdot \frac{d^{2} C}{d z^{2}}=0
$$

где $C$ - безразмерная концентрация, $z$ - безразмерная высота насадки, $P e=\frac{w \cdot 1_{0}}{D}-$ критерий Пекле, причем $w-$ линейная скорость потока, $1_{0}$ - определяющий линейный размер (в данном случае номинальный размер насадки), $D$ - коэффициент продольного перемешивания жидкости, учитывающий как турбулентную, так и молекулярную диффузию.

Связь между дисперсией и критерием Пекле в данном случае, учитывая отсутствие стока индикатора в застойную зону, выражается уравнением:

$$
\sigma^{2}=\frac{2}{P e^{2}}\left(P e-1+e^{-P e}\right),
$$

которое при достаточно высоком значении $P e$ практически дает $\sigma^{2}=2 / P e$.

В литературе приводятся эмпирические уравнения, выражающие зависимость критерия Пекле от критериев Рейнольдса и Галилея $\left[{ }^{1-4}\right]$ для различных случаев течения жидкости на насадке. С целью установить, кроме указанных критериев, влияние силы поверхностного натяжения (характеризуемого критерием Вебера) на продольное перемешивание нами изучалась корреляционная зависимость между критериями:

$$
P e=A \cdot R e^{m} \cdot G a^{n} \cdot W e^{p},
$$

где критерий Рейнольдса $R e=\frac{w \cdot 1_{0}}{\mu}$, критерий Галилея $G a=\frac{1_{0}^{3} \varrho^{2} g}{\mu^{2}}$ и критерий Вебера $W e=\frac{\varepsilon}{g 1_{0}^{2} \varrho}$. В этих критериях $\varrho-$ плотность, $\mu-$ вязкость, $\varepsilon-$ поверхностное натяжение, $g$ - ускорение свободного падения.

На основе экспериментальных данных проведены регрессионный и корреляционный анализы, в ходе которых по методу наименьших квадратов определялись числовые значения коэффициентов уравнения (3) и оценивалась корреляция между отдельными критериями. При этом выяснилось отсутствие статистически надежной корреляционной связи между критериями $P e$ и $W e$ (частный коэффициент корреляции в случае исключения влияния других критериев $\left.r_{P e W e} \cdot R e G a=0,30\right)$ несмотря на варьирование поверхностного натяжения жидкостей в довольно широких преде- 
лах (от 0,021 до 0,072 H/м). Следовательно, поверхностное натяжение не оказывает заметного влияния на продольное перемешивание, и критерий $W e$ можно исключить из уравнения (3). В опытах варьировались плотность орошения от 0,08 до $2,92 \mathrm{~m}^{3} / \mathrm{s}^{2} \cdot u$, вязкость жидкости от 0,27 до 2,40 спз и плотность жидкости от 680 до $1000 \mathrm{\kappa 2} / \mathrm{m}^{3}$.

Числовые значения коэффициентов уравнения (3), полученные в результате регрессионного анализа наших опытных данных, с доверительными границами, установленными с 95\%-ной вероятностью, следующие: $A=56,9 ; m=0,248 \pm 0,111$ и $n=-0,124 \pm 0,078$.

Широкие доверительные границы, указывающие на довольно сильное рассеивание экспериментальных данных, характерны и для работ других авторов. Аналогичные опыты, проведенные нами в ходе технологического процесса на полупромышленном гидрохлораторе, в основном подтвердили результаты, полученные на лабораторной установке.

Далее, задержка жидкости насадкой. Удерживающая способность объем жидкости в колонне, отнесенный к единице объема насадки определялась нами по среднему времени пребывания частиц жидкости в колонне:

$$
H=\frac{L \tau}{V},
$$

где $L$ - объемный поток жидкости в колонне, $V$ - объем насадки.

В литературе для определения удерживающей способности насадки приводятся корреляционные уравнения разного вида. Хорошую корреляцию наших данных (коэффициент множественной корреляции $R=0,82$ ) дает уравнение

$$
H=A^{\prime} \cdot R e^{m^{\prime}} \cdot G a^{n^{\prime}},
$$

принятое и другими авторами $\left[{ }^{5,6}\right]$. На основе наших результатов получены следующие числовые значения коэффициентов регрессионного уравнения (4) с 95\%-ными доверительными границами: $A^{\prime}=0,562 ; \quad m^{\prime}=$ $=0,122 \pm 0,055, n^{\prime}=-0,163 \pm 0,039$.

Еще более высокую корреляцию наших результатов $(R=0,87)$ показывает уравнение

$$
H=B \cdot F i^{1 / 3}+C,
$$

где $F i=F r / R e, F r=w^{2} / g \cdot 1$.

Это уравнение аналогично зависимости, выведенной Баченэном [7], если в ней член, выражающий влияние инерционных сил, заменить коэффициентом $C$, определяющим постоянную долю удерживающей способности. Отсутствие в данном случае заметного влияния инерционных сил выяснилось в ходе регрессионного анализа. Аналогичным образом выяснилось также отсутствие заметного влияния силы поверхностного натяжения (We). Коэффициенты уравнения (5) с 95-ными доверительными границами: $B=3,083 \pm 0,614$ и $C=0,0262$.

В опытах на полупромышленном гидрохлораторе было установлено, что значения удерживающей способности его примерно на $30 \%$ выше значений, получаемых по уравнению (5), несмотря на примерное геометрическое и гидродинамическое сходство процессов, проведенных в лабораторном и полупромышленном аппаратах. По-видимому, главную роль в этом сыграло различие в материале насадок у лабораторной и полупромышленной колонн. Физические свойства поверхностей обеих насадок (угол смачивания, шероховатость и адсорбционные свойства) довольно сильно отличались друг от друга. 
ЛИ Т Е Р А Т У Р A

1. Ot a ke T., Kun ug it a E., Chem. Eng. Japan, 22, 144 (1958).

2. S a ter V. E., L e ve n s p i e l O., Ind. Eng. Chem. Fundam., 5, 86 (1966).

3. Furzer I. A., Michell R. W., AIChE J., 16, 380 (1970).

4. P h a m Co, B i b a u d R., Can. J. Chem. Eng., 49, 727 (1971).

5. O t a ke T., O k a d a K., Chem. Eng. Japan, 17, 176 (1953).

6. Kunugit a E., Ot a ke T., Yosh i i K., Chem. Eng. Japan, 26, 672 (1962).

7. B u ch a n a n J. E., Ind. Eng. Chem. Fundam., 6, 400 (1967).

\section{Институт химии}

Академии наук Эстонской ССР
Поступила в редакцию $15 /$ X 1974

T. VOLENS, T. PETROVSKAJA, G. RAJALO

\section{PIKISEGUNEMINE JA VEDELIKU SALVESTUMINE TÄIDISKOLONNIS VÄIKESE KIIRUSEGA VOOLAVA GAASI KORRAL}

Artikkel käsitleb täidiskolonni pikisegunemise ja salvestumisvõime uurimise tulemusi, kui voolava gaasi kiirus on väike. Katseandmete analüüsi põhjal esitatakse vastavad kriteriaalvõrrandid.

T. VOLENS, T. PETROVSKAYA, G. RAJALO

\section{AXIAL MIXING AND HOLDUP IN RING-PACKED COLUMNS AT A LOW RATE OF THE GAS PHASE FLOW}

The paper deals with the results of studies of axial mixing and holdup in ring-packed columns at a low rate of the gas phase flow. As a result of the analysis of experimental data, the corresponding criterial equations have been determined. 